\title{
Nonlinear System Finite-Time Output Reachability for Flight Control given Uncertain Airspeed
}

\author{
Hossein Rastgoftar* \\ *Postdoctoral Research Fellow, Aerospace Engineering Department, 1320 Beal Avenue, Ann Arbor MI, USA. \\ Xiangyu $\mathrm{Ni}^{\dagger}$ \\ ${ }^{\dagger}$ MS Student, Aerospace Engineering Department, 1320 Beal Avenue, Ann Arbor MI, USA. \\ Ella M. Atkins ${ }^{\ddagger}$ \\ ${ }^{\ddagger}$ Professor, Aerospace Engineering Department, 1320 Beal Avenue, Ann Arbor MI, USA.
}

\begin{abstract}
This paper demonstrates stable level flight control given nonlinear dynamics in the presence of uncertainty in pitot tube airspeed measurements. It is first shown that a commanded cruise altitude can be reached in finite time by applying a recently-proposed finite-time reachability model. Then, the paper proposes a new dynamic decision making unit (DMU) to govern level flight in the presence of uncertain pitot tube readings. The DMU uses a Markov Decision Process to update the controls over a finite time-horizon, where MDP states are obtained from discretization of the output and MDP cost is proportional to the deviation from the desired output. A transition probability calculator learns MDP transition probabilities over time.
\end{abstract}

\section{Nomenclature}

\author{
$\alpha \quad$ Angle of Attack \\ $\bar{q} \quad$ Dynamic Pressure \\ $\beta \quad$ Side Slipe Angle \\ $\delta_{a} \quad$ Aileron Control Inputs \\ $\delta_{e} \quad$ Elevator Control Inputs \\ $\delta_{r} \quad$ Rudder Control Inputs \\ $\gamma \quad$ Discount factor \\ $\phi, \theta, \psi$ Roll, Pitch, Yaw Angle \\ $\rho \quad$ Air Density \\ $\mathbf{V}_{W} \quad$ Wind Velocity with Respect to Ground Resolved in Body Frame \\ $\mathbf{V}_{A C} \quad$ Aircraft Velocity with Respect to Ground Resolved in Body Frame \\ $b \quad$ Wingspan \\ c Wing Chord Length \\ $C_{D f} \quad$ Drag Coefficients \\ $C_{D g a}$ Drag Coefficients \\ $C_{D g e}$ Drag Coefficients \\ $C_{D g r} \quad$ Drag Coefficients \\ $C_{L f} \quad$ Lift Coefficients \\ $C_{l f} \quad$ Roll Moment Coefficients \\ $C_{\text {Lga }} \quad$ Lift Coefficients \\ $C_{\text {lga }} \quad$ Roll Moment Coefficients \\ $C_{\text {Lge }} \quad$ Lift Coefficients \\ $C_{\text {lge }} \quad$ Roll Moment Coefficients
}

* Postdoctoral Research Fellow, Aerospace Engineering Department, 1320 Beal Avenue, Ann Arbor MI, USA.

${ }^{\dagger}$ MS student, Aerospace Engineering Department, 1320 Beal Avenue, Ann Arbor MI, USA.

${ }^{\ddagger}$ Professor, Aerospace Engineering Department, 1320 Beal Avenue, Ann Arbor MI, USA. Please address all correspondence to this author. 


$\begin{array}{ll}C_{L g r} & \text { Lift Coefficients } \\ C_{l g r} & \text { Roll Moment Coefficients } \\ C_{m f} & \text { Pitching Moments Coefficients } \\ C_{m g a} & \text { Pitching Moments Coefficients } \\ C_{m g e} & \text { Pitching Moments Coefficients } \\ C_{m g r} & \text { Pitching Moments Coefficients } \\ C_{n f} & \text { Yaw Moment Coefficients } \\ C_{n g a} & \text { Yaw Moment Coefficients } \\ C_{n g e} & \text { Yaw Moment Coefficients } \\ C_{n g r} & \text { Yaw Moment Coefficients } \\ g & \text { Gravitational Acceleration } \\ I_{x x} & \text { Moment of Inertia } \\ I_{x z} & \text { Moment of Inertia } \\ I_{y y} & \text { Moment of Inertia } \\ I_{z z} & \text { Moment of Inertia } \\ m & \text { Aircraft Mass } \\ n_{a} & \text { Cardinality of the set of MDP AC } \\ p, q, r & \text { Aircraft Angular Velocity with Respect to Ground } \\ R & \text { Matrix of MDP cost } \\ S & \text { Wing Area } \\ T & \text { Throttle } \\ T P & \text { Transition probability tensor } \\ T P_{i} & \text { Transition probability matrix associated with the MDP action } i \in A C \\ U & \text { Control Input } \\ u, v, w & \text { Aircraft Velocity with Respect to Ground } \\ v_{w x} & x \text {-component of } \mathbf{V}_{W} \\ v_{w y} & y \text {-component of } \mathbf{V}_{W} \\ v_{w z} & z \text {-component of } \mathbf{V}_{W} \\ X & \text { Control State } \\ Y & \text { Control Output } \\ y, z & \text { Aircraft Position with Respect to Ground } \\ z_{d} & \text { Desired Altitude } \\ & \end{array}$

\section{Introduction}

Although pitot tube systems provide a reliable method to measure airspeed, failures of the pitot system have also led to a number of accidents for both transport and general aviation aircraft. ${ }^{1-3}$ Commercially-available autopilots typically recognize the pitot failures and disengage the autopilot in response, yet the combination of autopilot disconnect and crew reaction has led to incidents such as Air France Flight $447^{4}$ and Aeroperu Flight $603^{5}$ in which the ground crew covered the Pitot-static system with tape for cleaning but forgot to remove it before flight.

Future autopilot systems might override inappropriate automation/crew behaviors under such situations to increase the chance of survival. As shown in $^{6-8}$ one strategy is to rebuild sensor data based on the readings of some other functioning sensors. However, this strategy requires substantial online calculation and accurate aircraft dynamics models. For an uncertain system, a dual control method has been proposed. ${ }^{9-11} \operatorname{In}^{12-17}$ the Markov decision process (MDP) is also proposed for robust control. In $^{12}$ the optimal control is generated by using the MDP as an informationtheoretic approach for estimating dynamics. Robustness of controls with respect to uncertainties in the transition probability matrix is investigated by applying Temporal Logic Specifications in reference. ${ }^{18}$

The authors have recently proposed a finite-time reachability model (FTRM) used by multiple agents given linear dynamics for particles of a continuum. ${ }^{19}$ This paper extends the proposed FTRM to general nonlinear systems to assure reachability of a desired output in finite time. For the case study, we consider an airplane with a nonlinear model that aims to maintain level flight. It is shown how the desired output, defining desired altitude of the level flight, can be reached by the airplane in the absence of parameter uncertainty. We also propose a new dynamic decisionmaking model specifically to maintain level flight in the presence of parameter uncertainties with an uncertain pitot tube reading case study. The proposed dynamic decision making module (DDMM) augments a decision making unit (DMU) using the MDP to update controls (actions) executed by the flight dynamics in finite time. The DMU is also 
linked to a transition probability calculator (TPC) which estimates the probability of transitioning over the MDP states. Note that MDP states are determined by discretization of the output and MDP cost is proportional to the difference between actual and desired outputs.

This paper is organized as follows. In Section II notations and the formulation of the MDP are given. The primary problem of the paper is stated in Section III. Main elements of the DDMM and their functions are described in Section V. Simulation results are presented in Section VI followed by concluding remarks in Section VII.

\section{Markov Decision Process Background}

A Markov Decision Process (MDP) is defined by the tuple

$$
<S, A C, T P, R, \gamma>
$$

where $S$ is the set of MDP states with cadinality $n_{s}, A C$ is the set of MDP actions with cardinality $n_{a}, T P \in \mathbb{R}^{n_{s} \times n_{s} \times n_{a}}$ is the transition probability tensor, $R \in \mathbb{R}^{n_{s} \times n_{a}}$ is the matrix of MDP cost/reward values, and $\gamma$ is a discount factor. .Tensor $T P$ includes transition probability matrices $T P_{1}, T P_{2}, \ldots, T P_{n_{a}}$, where the matrix $T P_{i}$ defines probability of transition over MDP states under the MDP action $i \in A C$. The optimal policy is obtained from the Bellman equation, expressed as follows: ${ }^{20}$

$$
\pi^{*}(s)=\underset{a}{\operatorname{argmin}}\left\{R_{s, a}+\gamma \sum_{j=1}^{n_{s}} T P_{a s, j} V_{j}\right\}
$$

Note that $R_{s a}$ is the $s, a$ entry of the cost matrix $R(s \in S, a \in A C), T P_{a s, j}$ which denotes the $s, j$ entry of the matrix $T P_{a}(s, j \in S, a \in A C) . V_{j}$ is the $j$ th entry of the utility vector $V \in \mathbb{R}^{n_{s}}$.

\section{Problem Statement}

Consider a system with dynamics

$$
\begin{array}{r}
\dot{X}=F(X, U, \varpi) \\
Y=h(X)
\end{array}
$$

with control state $X \in \mathbb{R}^{n_{x}}$, control input $U \in \mathbb{R}^{n_{u}}$, and control output $Y \in \mathbb{R}^{n_{y}} . \varpi$ denotes parameter uncertainty of the system which is bounded but unknown. A goal of this work is for the system to learn uncertainty $\varpi$ over time and track the desired $Y_{d} \in \mathbb{R}^{n_{y}}$. For this purpose, a decision-making unit (DMU) augments the control system to update the control inputs over time by using the MDP. The DMU consists of two main parts: (i) a transition probability calculator (TPC), and (ii) a real-time decision maker. The TPC learns uncertainties by calculating the probability of transitions over MDP states based on available history of current control outputs as well as inputs (MDP actions) over a sliding time window. Given a transition probability matrix, the real-time decision maker specifies optimal control actions, where MDP cost is proportional to the difference between actual and desired control outputs.

This paper models the aircraft with nonlinear dynamics given by

$$
\dot{X}=F(X, U)
$$

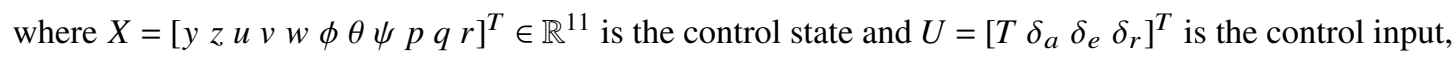

$$
F=\left[\begin{array}{c}
f_{1}(X, U) \\
\vdots \\
f_{11}(X, U)
\end{array}\right],
$$

The nonlinear expressions defining $F$ are specified below. 


$$
\begin{aligned}
f_{1} & =u \cos \theta \sin \psi+v(\sin \phi \sin \theta \sin \psi+\cos \phi \cos \psi)+w(\cos \phi \sin \theta \sin \psi-\sin \phi \cos \psi) \\
f_{2} & =-u \sin \theta+v \sin \phi \cos \theta+w \cos \phi \cos \theta \\
f_{3} & =v r-w q-g \sin \theta+\frac{T}{m}-\frac{\bar{q} S \cos \beta \cos \alpha}{m}\left(C_{D f}+C_{D g a} \delta_{a}+C_{D g e} \delta_{e}+C_{D g r} \delta_{r}\right) \\
& +\frac{\bar{q} S \sin \alpha}{m}\left(C_{L f}+C_{L g a} \delta_{a}+C_{L g e} \delta_{e}+C_{L g r} \delta_{r}\right) \\
f_{4} & =-u r+w p+g \sin \phi \cos \theta+\frac{\bar{q} S \sin \beta}{m}\left(C_{D f}+C_{D g a} \delta_{a}+C_{D g e} \delta_{e}+C_{D g r} \delta_{r}\right) \\
f_{5}= & u q-v p+g \cos \phi \cos \theta+\frac{\bar{q} S \cos \beta \sin \alpha}{m}\left(C_{D f}+C_{D g a} \delta_{a}+C_{D g e} \delta_{e}+C_{D g r} \delta_{r}\right) \\
& -\frac{\bar{q} S \cos \alpha}{m}\left(C_{L f}+C_{L g a} \delta_{a}+C_{L g e} \delta_{e}+C_{L g r} \delta_{r}\right) \\
f_{6}= & \frac{p \cos \theta+q \sin \theta \sin \phi+r \sin \theta \cos \phi}{\cos \theta} \\
f_{7}= & \frac{q \cos \theta \cos \phi-r \cos \theta \sin \phi}{\cos \theta} \\
f_{8}= & \frac{q \sin \phi+r \cos \phi}{\cos \theta} \\
f_{9}= & \frac{\bar{q} S b\left[\left(I_{z z} C_{l f}+I_{x z} C_{n f}\right)+\left(I_{z z} C_{l g a}+I_{x z} C_{n g a}\right) \delta_{a}+\left(I_{z z} C_{l g e}+I_{x z} C_{n g e}\right) \delta_{e}+\left(I_{z z} C_{l g r}+I_{x z} C_{n g r}\right) \delta_{r}\right]}{I_{x x} I_{z z}-I_{x z}^{2}} \\
& \frac{-I_{x z}\left(I_{y y}-I_{x x}-I_{z z}\right) p q-\left[I_{z z}\left(I_{z z}-I_{y y}\right)+I_{x z}^{2}\right] q r}{I_{x x} I_{z z}-I_{x z}^{2}} \\
f_{10}= & \frac{\bar{q} S c\left(C_{m f}+C_{m g a} \delta_{a}+C_{m g e} \delta_{e}+C_{m g r} \delta_{r}\right)-\left(I_{x x}-I_{z z}\right) p r-I_{x z}\left(p^{2}-r^{2}\right)}{I_{y y}-I_{x z}^{2}} \\
f_{11}= & \frac{\bar{q} S b\left[\left(I_{x z} C_{l f}+I_{x x} C_{n f}\right)+\left(I_{x z} C_{l g a}+I_{x x} C_{n g a}\right) \delta_{a}+\left(I_{x z} C_{l g e}+I_{x x} C_{n g e}\right) \delta_{e}+\left(I_{x z} C_{l g r}+I_{x x} C_{n g r}\right) \delta_{r}\right]}{I_{x x} I_{z z}-I_{x z}^{2}} \\
& \frac{-\left[I_{x x}\left(I_{y y}-I_{x x}\right)-I_{x z}^{2}\right] p q-I_{x z}\left(I_{x z}-I_{y r}\right.}{I_{x y}}
\end{aligned}
$$

The expression

$$
Y=\left[\begin{array}{l}
y \\
z
\end{array}\right]
$$

denotes the control output. For the below case studies the airplane is commanded to maintain a level flight at altitude $z_{d}$ given uncertainty in pitot tube readings. Mathematically speaking, the goal is for the airplane to track the desired output

$$
Y_{d}=\left[\begin{array}{c}
0 \\
z_{d}
\end{array}\right] .
$$

Note that in Eq. (3),

$$
\begin{gathered}
\bar{q}=\frac{1}{2} \rho\left(\mathbf{V}_{A C}-\mathbf{V}_{W}\right)^{2} \\
\sin \alpha=\frac{w-v_{w z}}{\sqrt{\left(u-v_{w x}\right)^{2}+\left(w-v_{w z}\right)^{2}}} \\
\cos \alpha=\frac{u-v_{w x}}{\sqrt{\left(u-v_{w x}\right)^{2}+\left(w-v_{w z}\right)^{2}}} \\
\sin \beta=\frac{v-v_{w y}}{\sqrt{\left(u-v_{w x}\right)^{2}+\left(v-v_{w y}\right)^{2}+\left(w-v_{w z}\right)^{2}}}
\end{gathered}
$$




$$
\cos \beta=\frac{\sqrt{\left(u-v_{w x}\right)^{2}+\left(w-v_{w z}\right)^{2}}}{\sqrt{\left(u-v_{w x}\right)^{2}+\left(v-v_{w y}\right)^{2}+\left(w-v_{w z}\right)^{2}}}
$$

\section{Finite-Time Reachability Model}

\section{IV.A. Linear System Preliminaries}

Consider a system with the dynamics

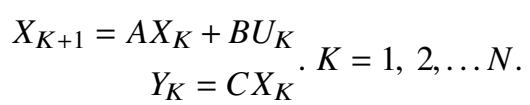

The dynamics (25) can be written in the following $l$-step-ahead form:

$$
X_{l}=A^{l} X+G_{l} Q_{l}
$$

where

$$
\begin{aligned}
& G_{l}=\left[\begin{array}{llll}
A^{l-1} B & \ldots & A B & B
\end{array}\right] \\
& Q_{l}=\left[\begin{array}{c}
U_{1} \\
\vdots \\
U_{l}
\end{array}\right] .
\end{aligned}
$$

Let desired outputs $Y_{d, 1}, Y_{d, 2}, \ldots, Y_{d, N+1}$ be known apriori, where

$$
Y_{1}=Y_{d, 1} .
$$

The first objective is to find $Q_{N}$ such that reachability of $Y_{d, N+1}$ is assured:

$$
\Omega_{R}=Y_{d, N+1}-Y_{N+1}=Y_{d, N+1}-C\left(A^{N} X_{1}+G_{N} Q_{N}\right)=0 .
$$

It is also desirable to minimize the cost

$$
\text { cost }=\sum_{j=1}^{N}\left(Y_{j}-Y_{d, j}\right)^{T} s\left(Y_{j}-Y_{d, j}\right)+Q_{N}^{T} R Q_{N}
$$

where $s \in \mathbb{R}^{n_{y} \times n_{y}}$ and $R \in \mathbb{R}^{\left(N n_{u} \times N n_{u}\right)}$ are positive definite weight matrices. These objectives are achieved, if $Q_{N}$ is chosen such that the augmented cost

$$
\operatorname{COST}=\sum_{j=1}^{N}\left(Y_{j}-Y_{d, j}\right)^{T} s\left(Y_{j}-Y_{d, j}\right)+Q_{N}{ }^{T} R Q_{N}+\lambda^{T} \Omega_{R}=Q_{N}{ }^{T}(R+P) Q_{N}+\left(M_{1}+M_{2}\right) Q_{N}+M_{3}+\lambda^{T} \Omega_{R}
$$

is minimized, where $\lambda \in \mathbb{R}^{n_{y}}$ is the Lagrange multiplier vector,

$$
\begin{gathered}
M_{1}=-G_{N}{ }^{T} C^{T} \lambda \in \mathbb{R}^{1 \times N . n_{u}} \\
M_{2}=2 \sum_{l=1}^{N} G_{l}^{T} C^{T} s\left(C A^{l} X_{1}-Y_{l}\right) \in \mathbb{R}^{1 \times N . n_{u}} \\
M_{3}=\sum_{l=1}^{N}\left\{\left(C A^{l} X_{1}-Y_{l}\right)^{T} s\left(C A^{l} X_{1}-Y_{l}\right)\right\}+\lambda^{T}\left(Y_{N+1}-A^{N} X_{1}\right) \in \mathbb{R} .
\end{gathered}
$$


Additionally, $P_{i j} \in \mathbb{R}^{n_{u} \times n_{u}}$ is the $i j$ block of the symmetric matrix $P$ and obtained as follows:

$$
P_{i j}=\sum_{l=\max \{i, j\}}^{N} B^{T}\left(A^{T}\right)^{l-i} s A^{l-j} B
$$

The optimal control $Q_{N}=Q_{N}{ }^{*}$ minimizing the COST in Eq. (13) is obtained from

$$
\frac{d \operatorname{COST}}{d Q_{N}}=0
$$

Therefore,

$$
Q_{N}^{*}=-\frac{1}{2}(R+P)^{-1}\left(M_{1}+M_{2}\right)=-\frac{1}{2}(R+P)^{-1}\left(-G_{N}^{T} C^{T} \lambda^{*}+M_{2}\right) .
$$

Substituting $Q_{N}{ }^{*}$ in reachability condition (11) results in

$$
\frac{1}{2} \Gamma \lambda^{*}=\frac{1}{2} C G_{N}(R+P)^{-1} M_{2}+Y_{N+1}-C A^{N} X_{1}
$$

or

$$
\lambda^{*}=\Gamma^{-1}\left(C G_{N}(R+P)^{-1} M_{2}+2 Y_{N+1}-2 H_{N} F_{N}\right)
$$

where

$$
\Gamma=C G_{N}(R+P)^{-1} G_{N}^{T} C^{T}
$$

is nonsingular. Therefore,

$$
Q_{N}^{*}=-\frac{1}{2}(R+P)^{-1}\left(-G_{N}^{T} C^{T}\left(\Gamma^{-1}\left(C G_{N}(R+P)^{-1} M_{2}+2 Y_{N+1}-2 H_{N} F_{N}\right)\right)+M_{2}\right) .
$$

\section{IV.B. Nonlinear Systems}

The nonlinear flight dynamics model presented in Section III is locally linearized as follows:

$$
\begin{array}{r}
X_{j+1}=A_{j} X_{j}+B_{j} U_{j} \\
Y_{j}=C X_{j}
\end{array}
$$

where

$$
\begin{gathered}
A_{j}=\frac{\partial F\left(X_{j}, U_{j}\right)}{\partial X_{j}} \\
B_{j}=\frac{\partial F\left(X_{j}, U_{j}\right)}{\partial U_{j}} \\
C=\left[\begin{array}{lllllllllll}
1 & 0 & 0 & 0 & 0 & 0 & 0 & 0 & 0 & 0 & 0 \\
0 & 1 & 0 & 0 & 0 & 0 & 0 & 0 & 0 & 0 & 0
\end{array}\right] .
\end{gathered}
$$

Let $j=(K-1) N+l(l=1,2, \ldots, N)$ be substituted in Eq. (24), where it is assumed that

$$
\begin{aligned}
& A_{(K-1) N+1}=A_{(K-1) N+2}=\cdots=A_{K N+1} \\
& B_{(K-1) N+1}=B_{(K-1) N+2}=\cdots=B_{K N+1} .
\end{aligned}
$$

Then, the flight dynamics can be expressed by $l$-step-ahead form,

$$
\begin{gathered}
X_{(K-1) N+l}=A_{(K-1) N+1}{ }^{l} X_{(K-1) N+1}+G_{K, l} Q_{K, l} l=1,2, \ldots, N, \\
Y_{(K-1) N+l}=C X_{(K-1) N+l} l
\end{gathered}
$$

where

$$
G_{K, l}=\left[\begin{array}{llll}
A_{(K-1) N+1}{ }^{l-1} B_{(K-1) N+1} & \ldots & A_{(K-1) N+1} B_{(K-1) N+1} & B_{(K-1) N+1}
\end{array}\right] \in \mathbb{R}^{11 \times(4 l)}
$$




$$
Q_{K, l}=\left[\begin{array}{c}
U_{(K-1) N+1} \\
\vdots \\
U_{(K-1) N+l}
\end{array}\right] . \in \mathbb{R}^{(4 l) \times 1}
$$

Given desired output $Y_{d, K N+1}$, the reachability condition becomes

$$
\Omega_{K, R}=Y_{d, K N+1}-Y_{K N+1}=Y_{d, K N+1}-C\left(A_{(K-1) N+1}{ }^{l} X_{K N}+G_{K, N} Q_{K, N}\right)=0 .
$$

The goal is to find $Q_{K, N}$ such that

$$
\begin{array}{r}
\operatorname{COST}=\sum_{l=1}^{N}\left(Y_{(K-1) N+l}-Y_{d,(K-1) N+l}\right)^{T} s\left(Y_{(K-1) N+l}-Y_{d,(K-1) N+1}\right)+Q_{K, N}{ }^{T} R Q_{K, N}+\lambda^{T} \Omega_{K, R}= \\
Q_{K, N}{ }^{T}\left(R+P_{K}\right) Q_{K, N}+\left(M_{K, 1}+M_{K, 2}\right) Q_{K, N}+M_{K, 3}+\lambda^{T} \Omega_{K, R}
\end{array}
$$

is minimized, where

$$
\begin{gathered}
M_{K, 1}=-G_{K, N}^{T} C^{T} \lambda \in \mathbb{R}^{4 N} \\
M_{K, 2}=2 \sum_{l=1}^{N} G_{K, l}^{T} C^{T} s\left(C A_{(K-1) N+1}{ }^{l} X_{(K-1) N+l}-Y_{(K-1) N+l}\right)
\end{gathered}
$$

$M_{K, 3}=\sum_{l=1}^{N}\left[\left(C A_{(K-1) N+1}{ }^{l} X_{(K-1) N+l}-Y_{(K-1) N+l}\right)^{T} s\left(C A_{(K-1) N+1}{ }^{l} X_{(K-1) N+l}-Y_{(K-1) N+l}\right)+\lambda^{T}\left(Y_{K N+1}-A_{(K-1) N+1}{ }^{N} X_{1}\right)\right.$.

The $i j$ block of the matrix $P_{K}$ is obtained from Eq. (17), where $A$ and $B$ are substituted by $A_{(K-1) N+1}$ and $B_{(K-1) N+1}$, respectively.

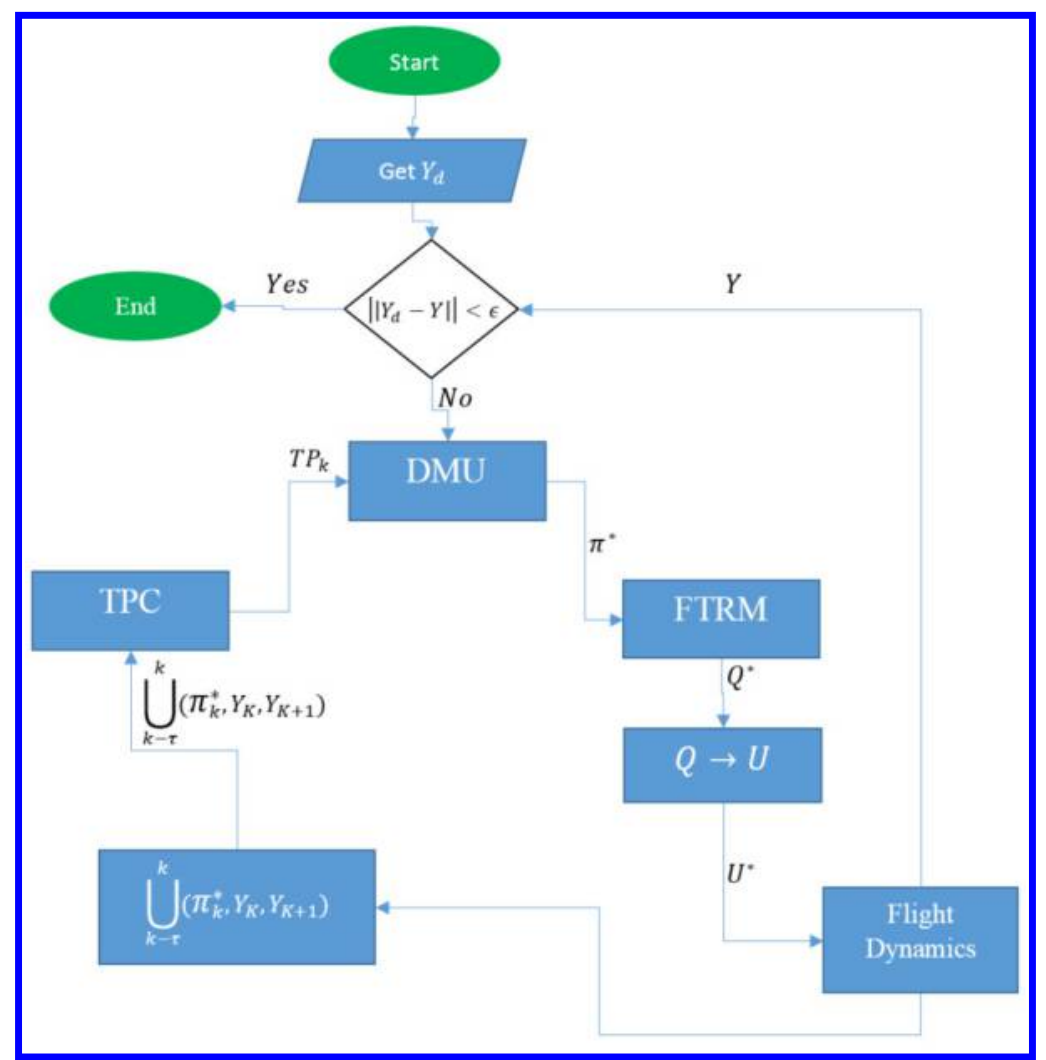

Figure 1: Structure of the Dynamic Decision Making Model (DDMM) 


\section{Dynamic Decision Making Model}

This section proposes a dynamic decision making model (DDMM) for achieving robust performance in the presence of uncertainty with unknown bounds. The structure of the DDMM is shown in Fig. 1. Components of the DDMM as well as their functions are described below.

\section{V.A. Main Components of DDMM}

As shown in Fig. 1, the decision-making unit (DMU), finite-time reachability model (FTRM), transition probability calculator (TPC), $Y \rightarrow s$ converter, $Q \rightarrow U$ converter and flight dynamics are the main components of the DDMM. Each is described below.

$Y \rightarrow s$ Converter: Let

$$
Y=\left[\begin{array}{c}
Y_{1} \\
\vdots \\
Y_{n_{y}}
\end{array}\right]
$$

be the control output, where $Y_{i} \in\left[Y_{i, \min }, Y_{i, \max }\right]\left(i=1,2, \ldots, n_{y}\right)$. Using

$$
\gamma_{i}=\left\lceil 5 \frac{Y_{i}-Y_{i, \text { min }}}{Y_{i, \text { max }}-Y_{i, \min }}\right\rceil
$$

output $Y$ is uniquely mapped to $\left(\gamma_{1}, \gamma_{2}, \ldots, \gamma_{n_{y}}\right)\left(\gamma_{i} \in\{1,2, \ldots, 5\}\right)$. Note that $\left(\gamma_{1}, \gamma_{2}, \ldots, \gamma_{n_{v}}\right)$ represents a hyper cuboid in $\mathbb{R}^{n_{y}}$. with the center located at

$$
C_{Y}=\left[\begin{array}{c}
Y_{1, \text { min }} \\
\vdots \\
Y_{n_{y}, \text { min }}
\end{array}\right]+\left[\begin{array}{c}
\left(\frac{1}{2}+\frac{\gamma_{1}-1}{5}\right)\left(Y_{1, \text { max }}-Y_{1, \text { min }}\right) \\
\vdots \\
\left(\frac{1}{2}+\frac{\gamma_{n_{y}}-1}{5}\right)\left(Y_{n_{y}, \text { max }}-Y_{n_{y}, \text { min }}\right)
\end{array}\right]
$$

Given $\left(\gamma_{1}, \gamma_{2}, \ldots, \gamma_{n_{y}}\right)$,

$$
s=\sum_{i=1}^{n_{y}-1} 5^{n_{y}-i}\left(\gamma_{i}-1\right)+\gamma_{n_{y}} \in S
$$

represents an MDP state, thus $n_{s}=|S|=5^{n_{y}}$ is the cardinality of the set of MDP states. Note that $\left(\gamma_{g, 1}, \ldots, \gamma_{g, n_{y}}\right)=$ $(3, \ldots, 3)$ is the hypercuboid representing the desired output providing the level flight condition. Therefore,

$$
s_{g}=2+\sum_{i=1}^{n_{y}-1} 5^{n_{y}-i}
$$

is the desired MDP state. As an example, consider the output of the flight dynamics,

$$
Y_{N(K-1)+1}=\left[\begin{array}{l}
y_{N(K-1)+1} \\
z_{N(K-1)+1}
\end{array}\right],
$$

where $n_{y}=2, y_{\min } \leq y \leq y_{\max }$ and $z_{\min } \leq z \leq z_{\max }$. Given $y_{\min }, y_{\max }, z_{\min }$, and $z_{\max }$,

$$
\begin{aligned}
& \gamma_{1}=\left\lceil 5 \frac{y_{N(K-1)+1}-y_{\min }}{y_{\max }-y_{\min }}\right\rceil \\
& \gamma_{2}=\left\lceil 5 \frac{z_{N(K-1)+1}-z_{\min }}{z_{\max }-z_{\min }}\right\rceil .
\end{aligned}
$$

As shown in Fig. 2, the output domain is partitioned into 9 tiles where each tile represents an MDP state.

$D M U$ : The decision making unit specifies optimal actions by using MDP. MDP states are obtained by discretization of the control output (see Eq.(32).) and are defined by the set

$$
S=\left\{1,2, \ldots, 5^{n_{y}}\right\} .
$$




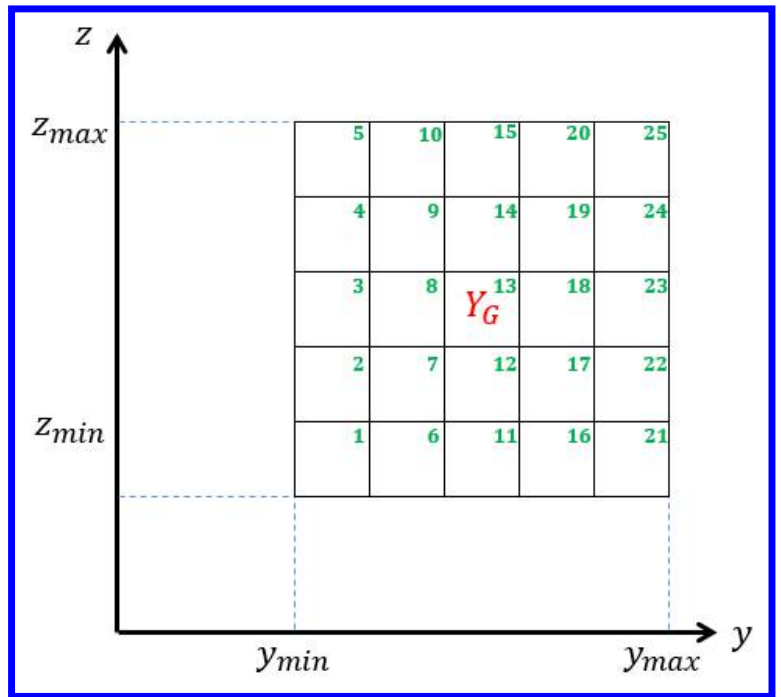

Figure 2: Schematic of output discritization of flight dynamics

By executing an MDP action $a_{j}$, the goal of output $Y_{N(K-1)+1}$ identified by $\left(\gamma_{1}, \ldots, \gamma_{n_{y}}\right)$ is to reach $Y_{d, N K+1}$ at the center of the hyper cuboid that is identified by $\left(\gamma_{1}+j_{1}, \ldots, \gamma_{n_{y}}+j_{n_{y}}\right)$. Note $j_{k} \in\{-1,0,1\}\left(k=1,2, \ldots, n_{y}\right)$, so there exist $n_{a}=3^{n_{y}}$ possible actions.

The transition probability matrix associated with an action $a_{j} \in A_{C}$ is determined based on empirical data as described in the sequel.

MDP cost is a metric to measure deviation from the goal state specified by $\left(\gamma_{g, 1}, \ldots, \gamma_{g, n_{y}}\right)=(3, \ldots, 3)$. Given $Y$, $\left(\gamma_{1}, \gamma_{2}, \ldots, \gamma_{n_{y}}\right)$ is obtained from Eq. (30), and MDP cost is defined by the matrix

$$
C=\mathbf{1}_{1 \times n_{a}} \otimes c \in \mathbb{R}^{n_{s} \times n_{s}},
$$

where the entry $s=\sum_{i=1}^{n_{y}-1} 5^{n_{y}-i}\left(\left(\gamma_{i}-1\right)+\gamma_{n_{y}}\right.$ of $c \in \mathbb{R}^{5^{n_{y}}}$ is given by

$$
c_{s}=\omega_{c} \sum_{i=1}^{n_{y}}\left(\gamma_{i}-2\right)^{2}
$$

and $\omega_{c} \in \mathbb{R}_{+}$is a scaling factor.

FTRM: Given $Y_{N(K-1)+1}$, the output at the time $N(K-1)+1,\left(\gamma_{1}, \gamma_{2}, \ldots, \gamma_{n_{y}}\right)$ is determined by using the Eq. (30). Knowing $Y_{N(K-1)+1}$ and MDP action issued by the DMU at the time $N(K-1)+1$, the desired output $Y_{d, K N+1}$ at the center of the target hyper cuboid is determined. By knowing $A_{N(K-1)+1}, B_{N(K-1)+1}, Y_{N(K-1)+1}$ and $Y_{d, K N+1}, Q_{K, N}$ is obtained from the Eq. (23).

TPC: It is assumed that the output history is sufficiently informative so the transition probability tensor associated with all MDP actions can be reasonably estimated based on the available history of output evolution. Let

$$
\bigcup_{j=1}^{\tau}\left(Y_{(K+1-j) N+1}, Y_{(K-j) N+1}, \pi^{*}{ }_{K-j}\right)
$$

denote the available history of the current and historical outputs as well as policies issued by the DMU during the time interval $[(K-\tau) N+1, K N+1]$. By knowing $Y_{(K-j) N+1}$ the corresponding MDP states $s_{(K+1-j) N+1}$ and $s_{(K-j) N+1}$ are calculated by using Eq. (31). Therefore, the row $s_{(K+1-j) N+1}$ of the transition probability matrix $T P_{\pi^{*}}$ is updated at time $(K+1-j) N+1$.

$Q \rightarrow U$ Converter: In the $Q \rightarrow U$ converter unit, $Q_{K, N}$ is a converted sequence of control inputs,

$$
U_{N(K-1)+1}, U_{N(K-1)+2}, \ldots, U_{N K}
$$

executed by the flight system during the time interval $[N(K-1)+1, K N]$.

Remark: If the DMU issues the action "stay", the target hyper cuboid at the time $K N+1$ is the same as the current hyper cuboid that is determined by using Eq. (30). However, $Q_{N} \neq 0$ if $Y_{N(K-1)+1} \neq Y_{d, K N+1}$. In other words, the control input $Q_{K, N}$ is actively executed when $Y_{N(K-1)+1} \neq Y_{d, K N+1}$.

Remark: $Q_{K N}$ is inevitably a nonzero vector if the DMU does not issue the action "stay". 


\section{Results}

In this simulation, an aircraft aims to maintain a level flight condition at the altitude of $2000 \mathrm{~m}$ in presence of uncertain wind speed. We consider nonlinear continuous-time aircraft model as given in Eqs. (3) and (4). Simulation parameters of the airplane are listed in Table 1.

Table 1: Aircraft Simulation Parameters - T-2 Aircraft ${ }^{21}$

\begin{tabular}{cc}
\hline Parameter & Value \\
\hline \hline$S$ & $0.5483 \mathrm{~m}^{2}$ \\
$b$ & $2.0876 \mathrm{~m}$ \\
$c$ & $0.2789 \mathrm{~m}$ \\
$m$ & $22.5 \mathrm{~kg}$ \\
$I_{x x}$ & $1.7992 \mathrm{~kg} \cdot \mathrm{m}^{2}$ \\
$I_{y y}$ & $5.7677 \mathrm{~kg} \cdot \mathrm{m}^{2}$ \\
$I_{z z}$ & $7.3946 \mathrm{~kg} \cdot \mathrm{m}^{2}$ \\
$I_{x z}$ & $0.1627 \mathrm{~kg} \cdot \mathrm{m}^{2}$ \\
\hline
\end{tabular}

By using the linearization model described in Section IV.B, the flight dynamics can be converted into the discrete form given in Eq. (8). It is assumed that wind velocity is uncertain but bounded. Also, $y_{\min }<y<y_{\max }, z_{\min }<z<$ $z_{\max }$, where $y_{\min }=-2.5 \mathrm{~m}, y_{\max }=2.5 \mathrm{~m}, z_{\min }=1987.5 \mathrm{~m}$ and $z_{\max }=2012.5 \mathrm{~m}$. Given control output

$$
Y=\left[\begin{array}{l}
y \\
z
\end{array}\right],
$$

$\gamma_{1}$ and $\gamma_{2}$ are obtained from Eq. (30),

$$
\begin{aligned}
& \gamma_{1}=\left\lceil\frac{5(y+2.5)}{5}\right\rceil \\
& \gamma_{2}=\left\lceil\frac{5(z-1987.5)}{25}\right\rceil .
\end{aligned}
$$

where $\gamma_{1}, \gamma_{2} \in\{1,2,3,4,5\}$. Note that

$$
s=5\left(\gamma_{1}-1\right)+\gamma_{2} \in S
$$

represents a tile with the center located at

$$
C_{Y}=\left[\begin{array}{l}
y_{\min }+\left(\gamma_{1}-0.5\right) \Delta y \\
z_{\min }+\left(\gamma_{1}-0.5\right) \Delta z
\end{array}\right],
$$

where

$$
\begin{aligned}
& \Delta y=\frac{y_{\max }-y_{\min }}{5}=1 \mathrm{~m} \\
& \Delta z=\frac{z_{\max }-z_{\min }}{5}=5 \mathrm{~m} .
\end{aligned}
$$

In the absence of parameter uncertainty, the desired output $Y_{d, K N+1}$ can be reached from $Y_{N(K-1)+1}$ by applying the finite-time reachability control described in Section IV. However, $Y_{d, K N+1}$ is not necessarily reached from $Y_{N(K-1)+1}$ if there exists uncertainty associated with flight parameters. Under this circumstance, $Y_{K N+1}$ is not necessarily equal to $Y_{K N+1}$ and the DMU finds the best possible action by using the MDP to reduce deviation from the desired output.

An MDP action is defined as a transition in the output $(y-z)$ plane from a point inside the current tile into the center of one of the adjacent tiles including diagonal motions. Therefore, $n_{a}=3^{2}=9$ is the cardinality of the set of MDP actions $A C$. Let the transformation from tile $\left(\gamma_{1}, \gamma_{2}\right)$ into the center of the adjacent tile $\left(\gamma_{1}+j_{1}, \gamma_{2}+j_{2}\right)$ be considered as the action $a_{j} \in A C$, where $j_{1}, j_{1}$ can be either $-1,0$, or 1 . Then, the MDP action is characterized by

$$
a_{j}=3\left(j_{1}+1\right)+j_{2}+1 \text {. }
$$


A Monte Carlo simulation with 200 iterations was performed to obtain the transition probability tensor $T P \in$ $\mathrm{R}^{25 \times 25 \times 9}$ associated with stochasticity of the uncertain wind. After calculating the transition probability tensor, we use traditional value iteration to find an optimal policy at time $N(K-1)+1$.

In the simulation, wind is considered to be stochastic with the components shown in the Fig.3. In Fig.4 components of actual and desired outputs are shown versus time. Notice that black dots show the values of $Y_{d,(k-1) N+1}(k=1,2, \ldots)$ issued by the DMU. Also, red dots are the values of the intermediate desired outputs, $Y_{d,(k-1) N+2}, Y_{d,(k-1) N+3}, \ldots$, $Y_{d, K N}(k=1,2, \ldots)(k=1,2, \ldots)$. Moreover, blue curves illustrate components of actual output versus time. Observe that the difference between actual and desired outputs ultimately vanishes.

$$
Y_{G}=\left[\begin{array}{c}
0 \\
2000
\end{array}\right] \text {. }
$$
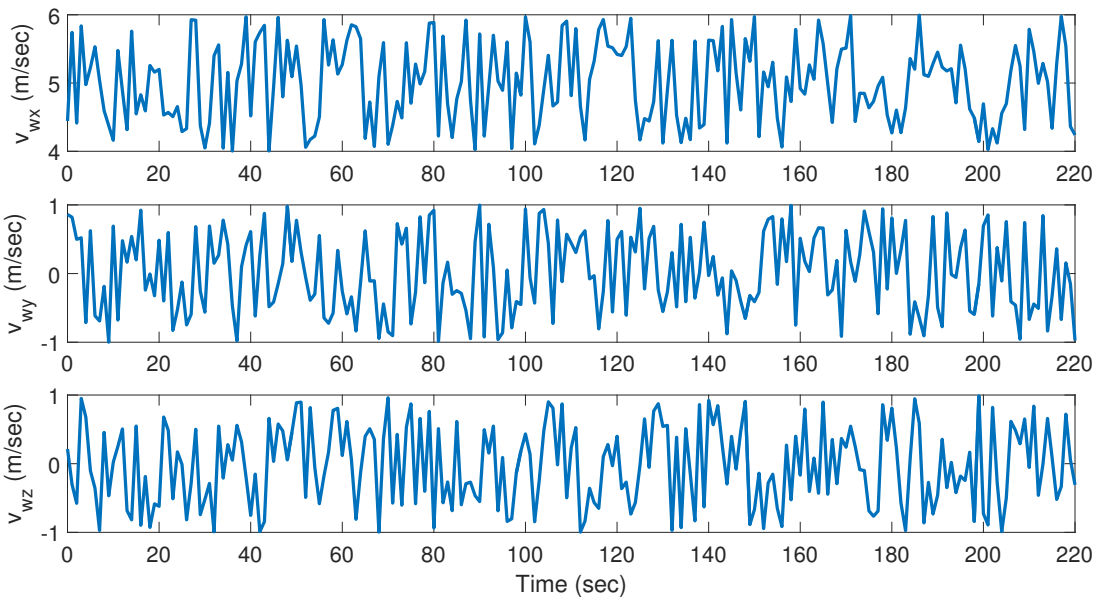

Figure 3: History of Wind Speed

The history of MDP actions and control inputs $T, \delta_{a}, \delta_{e}$, and $\delta_{r}$ are shown in Figs. 5 and 6, respectively. Notice that all simulation results above are based on a case where the mean value of $v_{w x}$ is $5 \mathrm{~m} / \mathrm{s}$. 


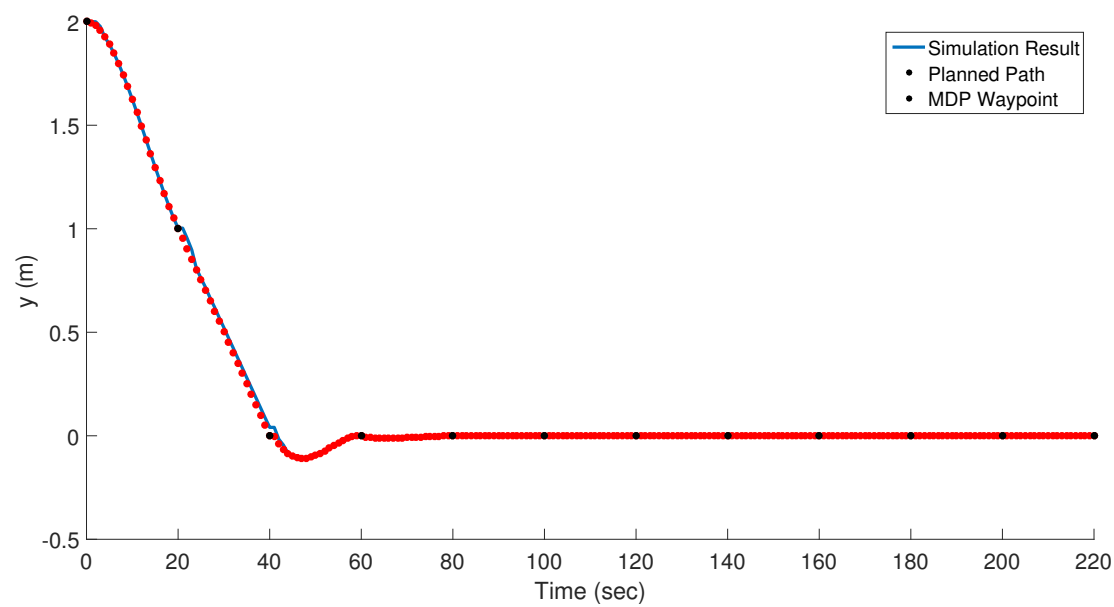

(a) y Position Versus Time

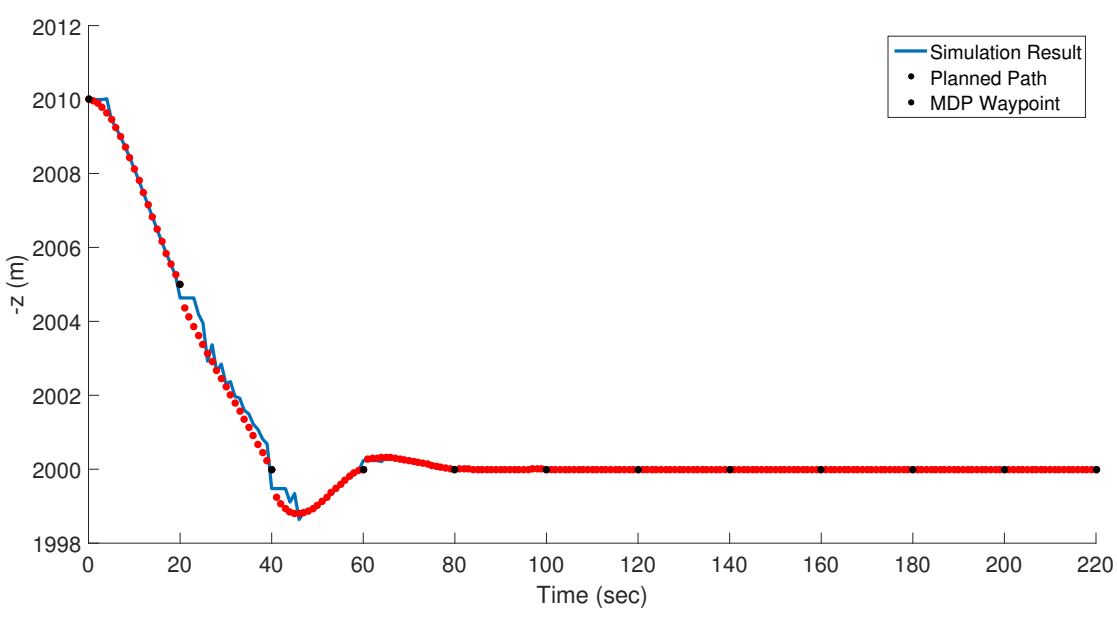

(b) $z$ Position Versus Time

Figure 4: Simulated History of All Control States

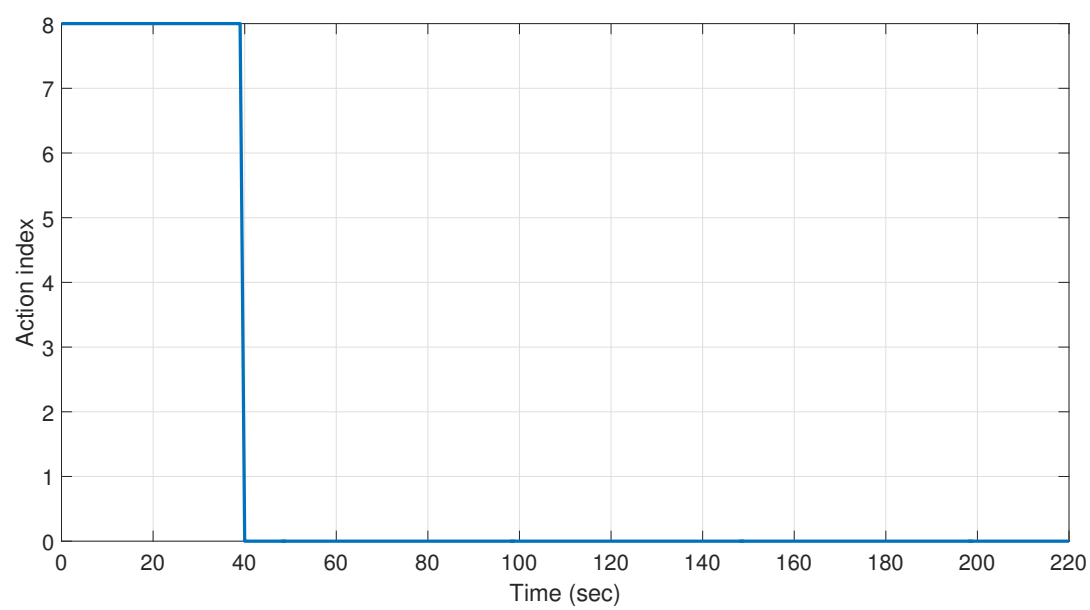

Figure 5: History of MDP Action Index 


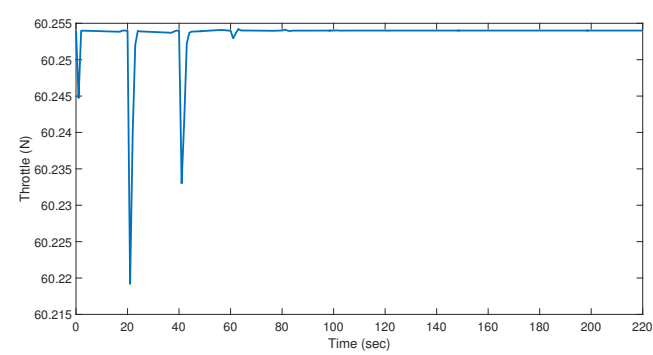

(a) Throttle Versus Time

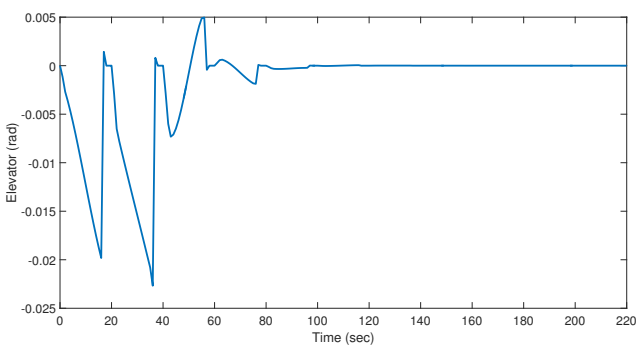

(c) Elevator Versus Time

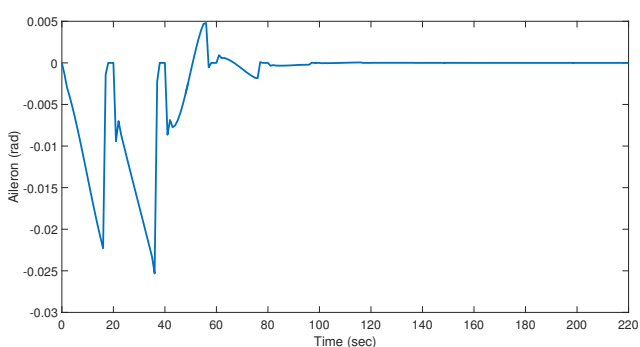

(b) Aileron Versus Time

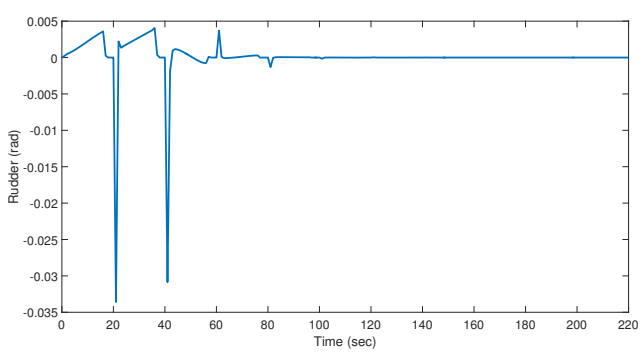

(d) Rudder Versus Time

Figure 6: History of Control Inputs 


\section{Conclusion}

In this paper, finite time reachability of the desired output is demonstrated. A dynamic decision-making model is proposed for real time control of a nonlinear system in the presence of parameter uncertainties. A case study was examined in which an aircraft maintains level flight at a desired altitude given uncertainty associated with pitot tube readings. We showed how the DMU augmenting the control system can apply the MDP to update controls (actions) executed by the airplane such that MDP cost, defined proportional to the difference between desired and actual outputs of the flight dynamics, is minimized. The transition probability calculator linked to the DMU estimates probability of transition over MDP states, where MDP states are determined by discretization of the control outputs.

\section{Acknowledgement}

This work was supported in part under Office of Naval Research (ONR) grant N000141410596.

\section{References}

\footnotetext{
${ }^{1}$ United States National Aeronautics and Space Administration, Federal Aviation Administration, Aviation Safety Reporting System (ASRS) Database, June 2016, http://asrs.arc.nasa.gov/search/database.html.

${ }^{2}$ United States Federal Aviation Administration, Incident Data Records, Aviation Safety Information Analysis and Sharing: Accident/Incident Data System (AIDS), June 2016, http://www.asias.faa.gov.

${ }^{3}$ Flight Safety Foundation, Aviation Safety Network, Accident Descriptions ASN Aviation Safety Database, June 2016, http://aviationsafety.net/database/.

${ }^{4}$ B. d'Enquetes, "Final report on the accident on 1st june 2009 to the airbus a330-203 registered f-gzcp operated by air france flight af 447 rio de janeiro-paris," Ministère de l'Écologie. du Dévéloppement durable, des Transports et du Logement, Paris, 2012.

${ }^{5}$ Aviation Safety Network, Aircraft Safety Database, Boeing 757-23A N52AW Lima, Peru, June 2016, http://aviationsafety.net/database/record.php?id=19961002-0.

${ }^{6}$ G. Ducard, K. Rudin, S. Omari, and R. Siegwart, "Strategies for sensor-fault compensation on uavs: Review, discussions \& additions," in Control Conference (ECC), 2014 European. IEEE, 2014, pp. 1963-1968.

${ }^{7}$ R. H. Chen and J. L. Speyer, "Sensor and actuator fault reconstruction," Journal of Guidance, Control, and Dynamics, vol. 27, no. 2, pp. 186-196, 2004.

${ }^{8}$ N. Tanaka, S. Suzuki, K. Masui, and H. Tomita, "Restructurable guidance and control for aircraft with failures considering gust effects," Journal of guidance, control, and dynamics, vol. 29, no. 3, pp. 671-679, 2006.

${ }^{9}$ Y. Cheng, S. Haghighat, and S. Di Cairano, "Robust dual control mpc with application to soft-landing control," in American Control Conference (ACC), 2015. IEEE, 2015, pp. 3862-3867.

${ }^{10} \mathrm{~A}$. Weiss and S. Di Cairano, "Robust dual control mpc with guaranteed constraint satisfaction," in Decision and Control (CDC), 2014 IEEE 53rd Annual Conference on. IEEE, 2014, pp. 6713-6718.

${ }^{11}$ T. Alpcan, "Dual control with active learning using gaussian process regression," arXiv preprint arXiv:1105.2211, 2011.

${ }^{12}$ T. Alpcan and I. Shames, "An information-based learning approach to dual control," 2015.

${ }^{13}$ E. M. Wolff, U. Topcu, and R. M. Murray, "Robust control of uncertain markov decision processes with temporal logic specifications," in Decision and Control (CDC), 2012 IEEE 51st Annual Conference on. IEEE, 2012, pp. 3372-3379.

${ }^{14}$ L. El Ghaoui and A. Nilim, "Robust solutions to markov decision problems with uncertain transition matrices," Operations Research, vol. 53, no. $5,2005$.

${ }^{15}$ J. A. Bagnell, A. Y. Ng, and J. G. Schneider, "Solving uncertain markov decision processes," 2001.

${ }^{16}$ M. L. Puterman, Markov decision processes: discrete stochastic dynamic programming. John Wiley \& Sons, 2014.

${ }^{17}$ C. Boutilier, T. Dean, and S. Hanks, “Decision-theoretic planning: Structural assumptions and computational leverage," Journal of Artificial Intelligence Research, vol. 11, no. 1, p. 94, 1999.

${ }^{18}$ H. Barringer, R. Kuiper, and A. Pnueli, "Now you may compose temporal logic specifications," in Proceedings of the sixteenth annual ACM symposium on Theory of computing. ACM, 1984, pp. 51-63.

${ }^{19}$ H. Rastgoftar and E. M. Atkins, "Continuum deformation of multi-agent systems under directed communication topologies," ASME Journal of Dynamic System Measurement and Control, 2016.

${ }^{20}$ S. Russell, P. Norvig, and A. Intelligence, "A modern approach,” Artificial Intelligence. Prentice-Hall, Egnlewood Cliffs, vol. 25, p. $27,1995$.

${ }^{21}$ J. A. Grauer and E. A. Morelli, "A generic nonlinear aerodynamic model for aircraft," in Proceedings of AIAA Atmospheric Flight Mechanics Conference, 2014, pp. 2014-0542.
} 